ORIGINAL ARTICLE

\title{
Scale of protection and the various types of sports mouthguard
}

\author{
D G Patrick, R van Noort, M S Found
}

Br J Sports Med 2005;39:278-281. doi: 10.1136/bjsm.2004.012658

See end of article for authors' affiliations

....................

Correspondence to: Dr David George Patrick, University of Sheffield, Department of Oral Health and Development, School of Clinical Dentistry, Claremont Crescent Sheffield S10 2TA, UK d.g.Patrick@sheffield.ac.uk

Accepted 20 July 2004
Objectives: To present a grading system of the protection offered by various types of mouthguard, together with an indication of associated risks, in order to make athletes aware of the consequences of improper or no mouth protection.

Methods: A review of the literature on mouthguards, mouthguard materials, and novel laminates for mouthguards was carried out as it was apparent that information on mouthguards was lacking. Studies on stock (or unfitted) mouthguards, mouth formed (boil and bite) mouthguards, and custom mouthguards are reviewed.

Results: A scale of protection offered by various mouthguards and novel laminates has been produced, where 0 indicates no mouthguard and 10 indicates a custom made mouthguard offering excellent protection.

Conclusions: From work carried out on laminates and the manufacturing processes of mouthguards, it became apparent that information was lacking to enable athletes make informed decisions about the best oral protection for their chosen sport.
M outhguards have been worn by sportsmen for almost a hundred years and were initially used by boxers. A mouthguard made from a piece of natural rubber that had been trimmed and hollowed out so that it would fit over the maxillary dentition, was worn to prevent chipped or broken teeth resulting from blows to the head. As it was not adapted to the teeth, the jaw had to be clenched to hold the mouthguard in place, making it difficult for the wearer to breathe. ${ }^{12}$ This type of unfitted mouthguard can still be bought today, although the materials have changed, ethylene vinyl acetate (EVA) being substituted for rubber. Most sports shops sell them and, surprisingly, they are sometimes recommended to sportsmen and women by their dentists. This type of mouthguard offers a very low level of protection to the wearer; it also has the added danger of the possibility that it may become dislodged and obstruct the air passage causing asphyxiation. Sportsmen should be actively discouraged from wearing such a mouthguard. ${ }^{3}$

Previous work on the efficacy of mouthguards and their use, and the compliance of athletes with rulings concerning their use during training and actual play, has recommended the wearing of custom made mouthguards, advising that stock mouthguards should not be worn. ${ }^{4}$ The wearing of a properly designed and fitted mouthguard to prevent oral trauma has been recognised as the best option. ${ }^{5}$ Brittle lacquer tests $^{6}$ have shown the effect that impact forces transmitted through mouthguards can have on teeth, and the fact that wearing a mouthguard can prevent knock outs and repetitive concussion is now gaining more credence, years after it was initially reported. ${ }^{7}$ Work carried out on the protective capabilities and physical responses to an impact of standard mouthguard material (EVA) and novel laminates for use in mouthguards has shown the levels of protection that are present. ${ }^{8}$ The processing of EVA during the manufacturing process has also been indicated as a factor to be accounted for in any testing regimen that is to correctly assess the physical characteristics of a thermo-formed material. ${ }^{9}$ To make athletes participating in contact sports, where the incidence of oral trauma or concussion is high, more aware of the level of protection that a mouthguard may offer, a grading system of the various types of mouthguard and their protection capabilities has been developed.

\section{CLASSIFICATION OF MOUTHGUARDS}

Three types of mouthguard are generally available: stock mouthguards, mouth formed mouthguards, and custom made mouth guards.

\section{Stock mouthguards}

Stock mouthguards come in different sizes, are ready to use, and are mostly made from either polyvinyl chloride (although the use of PVC for mouthguards has now been outlawed by the EU), polyurethane, or a co-polymer of vinyl acetate or ethylene. Generally, stock mouthguards are thought to be the least favourable as they offer the minimum protection and may even be thought of as dangerous as they may give a rugby player, for example, a false sense of security. ${ }^{4}$

\section{Mouth formed mouthguards}

Mouth formed mouthguards are known as "boil and bite" mouthguards, where a thermoplastic rim is heated in hot water then placed in the mouth and moulded by biting and sucking. These mouthguards have a poor fit on the teeth and tend to be thin over prominent teeth that are prone to damage.

\section{Custom made mouthguards}

Custom made mouthguards are made in a dental laboratory on a cast taken from an impression supplied by a dentist. A thermoplastic material is heated in a pressure or vacuum forming machine and when soft enough is placed over the cast and air pressure or a vacuum is applied which closely adapts the soft material to the cast.

Of the types listed it is generally thought that the custom made mouthguards are the best and offer the most protection. ${ }^{5}$

Abbreviations: EVA, ethylene vinyl acetate 


\section{MOUTHGUARD ASSESSMENT}

Mouthguards and the materials from which they have been made have generally been tested in the same way over the years. There have been many comparative studies of the various types of mouthguard and mouthguard materials. However, typical tests on the material do not really reveal the ideal properties being sought in a mouthguard material. Tests on mouthguards placed on a model or on the maxilla of a cadaver (Hickey et $a l^{7}$ ) may give a clearer indication of the protection offered by the mouthguard and the mouthguard material.

Hoffmann et $a l^{10}$ studied the mechanical and physical properties of several commercially available mouthguards. Mouthguards were fitted onto a specially made model so that tooth deflection caused by an impact from a pendulum ram could be recorded. Data from the teeth protected with a mouthguard were compared to data from unprotected teeth and it was found that the cushioning effects of the mouthguards were directly correlated to their thickness and that the force distribution was governed by the rigidity of the mouthguard. Oikarinen et al $l^{11}$ compared the "guarding capacity" of several mouth protectors whilst on a standard sized maxillary plaster model. A dropweight impact tester was constructed with the falling weight designed to simulate an ice hockey puck and mouthguards were constructed from two layers of material with a resilient layer next to the teeth. Using stepwise regression analysis, the only variable that had any statistical significance on the guarding capacity was the thickness of the soft layer next to the teeth.

To determine the effect of mouthguards on pressure changes and bone deformation within the skull, Hickey et $\mathrm{al}^{7}$ constructed an impact producing mechanism that was attached to an American football helmet so that a blow of known force could be delivered to the chin of an intact male cadaver. They did not examine the design of the mouthguard or the material from which it was made but did examine the protection capabilities of mouthguards as regards concussion.

Kim and Mathieu ${ }^{12}$ studied the lamination of mouthguards using finite element analysis. A flat ended indentor and a disc representing a colliding object were produced so that the stress distribution within mouthguard materials could be recorded. The tested laminates consisted of a hard and a soft material with a bi-laminated structure rather than a sandwich panel or a multi-layered structure. When the soft layer was uppermost (in contact with the indentor) there was no significant difference compared to a monolithic test piece. However, when the hard layer was positioned uppermost there was a significant effect on stress distribution, which effect could be increased by controlling the ratios of modulus and volume fractions of the top and bottom layers.

A visco-elastic polyurethane, Sorbothane, used in orthopaedic and sports applications because of its shock absorbing properties, was tested by Bulsara and Matthew ${ }^{13}$ as an intermediate layer between two layers of EVA. A piezoelectric transducer was used to measure the peak force transmitted through samples with and without the Sorbothane layer from a free falling steel ram. Bulsara and Matthew concluded that using an intermediate layer of Sorbothane may significantly dissipate the force of impact from a blow to the teeth and jaws.

In an attempt to develop a standard test procedure for mouthguard assessment, Greasley and Karet $^{14}$ and Greasley et $a l^{15}$ constructed an upper jaw made from a rubber arch containing replaceable ceramic teeth and a renewable composite jawbone on which mouthguards were to be tested. Different projectiles at various energies were impacted on to the model jaw by dropping them down a clear plastic tube whilst a mouthguard was in situ and damage to the teeth and jaw was recorded. The objective of the exercise was to produce a testing regime that could easily be applied to any mouthguard fitting the standard model they had made.

Westerman et al ${ }^{16}$ used an impact test rig similar to a Charpy or Izod impact machine and fitted with a blunt striker on the pendulum. Tests showed that the force transmitted through the mouthguard material was inversely related to the thickness of the material and that a small reduction in thickness of $1 \mathrm{~mm}$ resulted in an increase in transmitted force of $34 \%$. Westerman et $a l^{17}$ also assessed the energy absorption properties of a material containing pockets of air. It was reported that the inclusion of air cells within an EVA copolymer mouthguard material produced a reduction in transmitted forces when the impact was less than $10 \mathrm{kN}$.

Further to their earlier work, Godwin and Craig ${ }^{6}$ examined the stress transmitted through mouth protectors. Brittle lacquer coatings on maxillary models that were then fitted with mouthguards demonstrated quite graphically the effectiveness of the individual mouthguards.

Physical and mechanical tests were employed to examine the basic properties of 57 different mouthguard products by Going et $\mathrm{l}^{18}$ in 1974 . As well as determining material property tests for impact, energy absorption and resistance to impact penetration tests were performed using a rebound pendulum method. It was concluded that the dynamic energy data from the rebound test should be interpreted cautiously and that a high energy absorption level does not necessarily mean maximum protection, since some of the absorbed energy may be transmitted directly to the underlying tooth structure.

\section{MOUTHGUARD PROPOSAL}

It is proposed that mouthguards should have a composite laminate construction with a very compliant centre region and a more rigid outer layer, such as EVA (fig 1). In this way, a reduced impact force will be transferred to the teeth due to the shock absorbing capability of the compliant material layer. Harmful rebound energy will also be reduced as the composite laminate will return to its original shape more slowly than a single material system. ${ }^{8}$ Combinations of compliant/rigid materials could be built up in a multi-layered composite system with materials and layer thicknesses being adapted according to particular requirements.

During the course of this research the effect of heat processing on the physical properties of EVA was observed. Heat treatment reduced the process stresses, resulting in reduced impact force and increased displacement. The mouthguard manufacturing process, therefore, may compromise the performance of EVA as a protective device, the increased displacement being a real cause for concern. ${ }^{9}$

\section{GRADES OF PROTECTION}

Table 1 lists the suggested order of a scale of protection offered by various mouthguards.

Not wearing a mouthguard (grade 0 ) clearly offers the lowest protection for the obvious reasons of teeth being knocked out, lacerations to the lips, chipped teeth, and concussion.

A stock mouthguard (grade 1) of the type that has to be clenched between the teeth to stay in the mouth is ranked next. As previously stated, these mouthguards are dangerous and should not be worn as they instil a false sense of security in the wearer, are easily dislodged, and may present a choking hazard.

Boil and bite mouthguards (grade 2 ) are generally regarded as being little better than stock mouthguards. The fitting process may not result in a good, close fit. Also, if the mouthguard is bitten too hard during the fitting process, the occlusal thickness may be reduced sufficiently to compromise the already limited protection. 


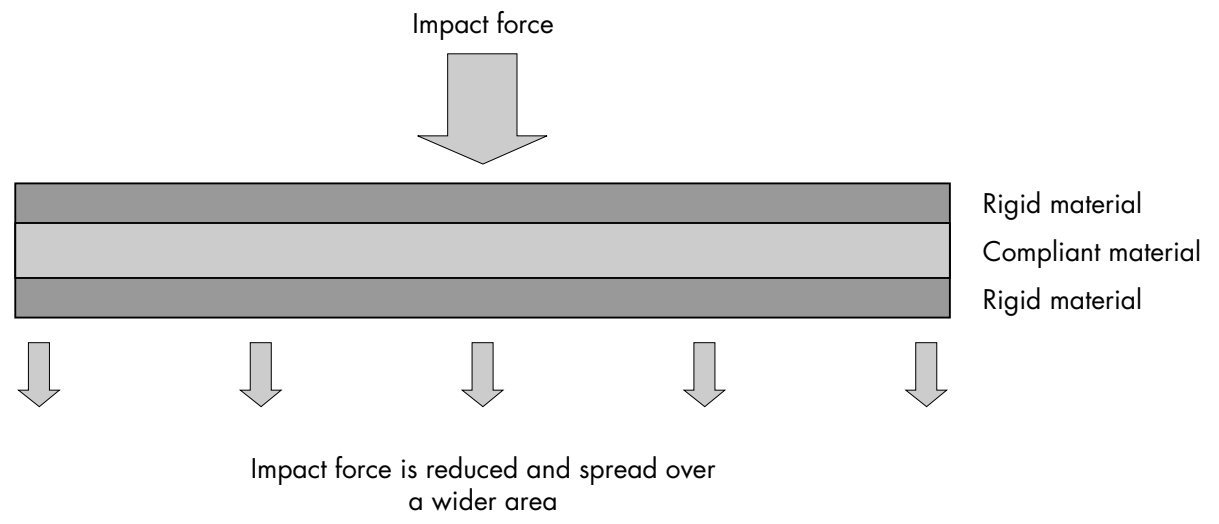

Figure 1 Distribution of force impacting on mouthguard with outer layers made of EVA.

An old custom made mouthguard $>5$ years old (grade 3 ) will have lost much of its initial properties and therefore will not be effective enough in the event of an impact. Such mouthguards become hard, may wear down on the occlusal surface, and become too thin to prevent concussion. They may also not fit very well after a few years if teeth have been moved, worn down, or restored.

Mouthguards between 2 and 5 years old (grade 4) may suffer some or all of the same problems of mouthguards $>5$ years old depending on the amount of use and/or abuse they have received and how much the wearer's dentition has changed.

A custom made mouthguard (grade 6) offers the best protection against trauma and concussion. However, it must be correctly made and conform to certain guidelines in relation to thickness and coverage of the dentition; if the mouthguard is too thin (grade 5) there will be insufficient thickness to offer adequate protection.

The following criteria should apply to mouthguards used in contact sports ${ }^{19}$ :

1. They should enclose the maxillary teeth to the distal surface of the second molars.

2. Thickness should be $3 \mathrm{~mm}$ on the labial aspects, $2 \mathrm{~mm}$ on the occlusal aspect, and $1 \mathrm{~mm}$ on the palatal aspect.

3. The labial flange should extend to within $2 \mathrm{~mm}$ of the vestibular reflection.

4. The palatal flange should extend about $10 \mathrm{~mm}$ above the gingival margin.

5. The edge of the labial flange should be rounded in cross section whereas the palatal edge should be tapered.

\begin{tabular}{|cl}
\hline Table 1 & Grades of protection \\
\hline Grade & Feature \\
\hline 10 & Ultimate aim: grades 8 and 9 combined \\
9 & Passed effective instrumented test to evaluate mouthguards \\
8 & Custom made mouthguard using improved design and \\
7 & materials \\
6 & Custom made mouthguard using improved materials \\
5 & Cund new custom made mouthguard \\
4 & Old custom made mouth mouth of insurfficient thickness \\
3 & Old custom made mouthguard, $>5$ years old \\
2 & Boil and bite mouthguard \\
1 & Stock mouthguard \\
0 & No mouthguard \\
\hline
\end{tabular}

6. When a maxillary guard is constructed it should be articulated against the matching mandibular model for optimum comfort.

However, as regards point 4 above, the palatal flange would be more easily tolerated if it was kept to a minimum: a palatal flange of $1-2 \mathrm{~mm}$ beyond the gingival margin would be ideal.

A mouthguard conforming to the above guidelines will provide maximum protection against oral trauma such as broken or avulsed teeth, soft tissue lacerations, and concussion. However, mouthguard materials, whilst effective, could be improved. The ideal mouthguard material is composed of a polyvinyl acetate-polyethylene mixture in the range of 18$24 \%$ polyvinyl acetate. ${ }^{21}$ Materials in this range exhibit a dynamic energy absorption of between 30.43 and $31.18 \mathrm{~mJ}$. After taking into account other factors such as tear strength $(41.7 \mathrm{~kg} / \mathrm{cm})$, water absorption $\left(0.106 \mathrm{mg} / \mathrm{cm}^{2}\right)$, static energy absorption $(7.14 \mathrm{~mJ})$, and elastic gradients, a material containing $18 \%$ polyvinyl acetate is recommended for mouthguards. ${ }^{21}$

Three areas that can be developed to improve custom made mouthguards:

1. Improved materials (grade 7) that are better at absorbing repeated impacts and transfer less energy to the teeth, jaws, and brain.

2. Improved mouthguard design (grade 8). Mouthguard design has not changed for years and is limited by the parameters, but the most at risk teeth need to be properly protected and the risk of repeated concussion negated.

3. An effective, instrumented, test for mouthguards (grade 9) is required so that new mouthguards can be tested. ${ }^{20}$ This test must be instrumented so that a quantifiable index of protection can be developed.

By combining items $1-3$ above, we can obtain the ultimate mouthguard (grade 10).

\section{DISCUSSION}

Mouthguards differ and wearers need to be aware of their various protective capabilities. Athletes are probably know that a custom made mouthguard is the best option, but in many instances price determines which mouthguard is used. Custom made mouthguards cost around $£ 25$, while a stock or boil and bite mouthguard costs around $£ 2-£ 6$, depending on the retailer. A grading system ranking mouthguards from 0 to 10 would allow a more informed choice, so that fewer poorly rated mouthguards would eventually be used and most would be custom made. Only through education and awareness of the efficacy of mouthguards can athletes make 


\section{What is already known on this topic}

Three types of mouthguard are generally available to protect against concussion and oral trauma. Of these, the custom made mouthguard offers the best protection.

an informed choice and opt for the best mouthguard available. The mouthguard must be comfortable and easy to wear, an aim achievable if guidelines for size and shape are followed. In addition, if a wide range of colours were available, then athletes could wear a mouthguard in their chosen team colour. It seems strange that though athletes are prepared to pay extraordinary sums of money for the latest kit, shoes, bat, racquet, etc, so little thought is given to protecting the teeth, mouth, and head from repeated concussion.

\section{CONCLUSION}

A grading system for sports mouthguards, indicating the risks associated with not wearing a mouthguard, is suggested. This would highlight the potential for trauma, allow mouthguards to be easily and quickly assessed, and would influence the type of mouthguard chosen. Over time it is hoped that the use of stock mouthguards would diminish in favour of the custom made mouthguard thereby reducing the incidence of oral trauma in contact sports.

\section{Authors' affiliations}

D G Patrick, R van Noort, M S Found, University of Sheffield, Sheffield, UK

Competing interests: none declared.

\section{REFERENCES}

1 Chapman PJ. The bimaxillary mouthguard: increased protection against orofacial and head injuries in sport. Aust J Sci Med Sport 1985;17:25-8.

2 Flanders RA. Mouthguards and sports injuries. Illinois Dent J 1993;62(1):13-6.

3 Chapman PJ. Orofacial injuries and the use of mouthguards by the 1984 Great Britain Rugby League touring team. Br J Sports Med 1985;19(1):34-6.
What this study adds

A grading system for sports mouthguards is suggested that would allow mouthguards to be easily and quickly assessed.

4 Widmer RP. The normal development of teeth. Aust Fam Physician 1992;21(9): 1251-61.

5 DeYoung AK, Robinson E, Godwin WC. Comparing comfort and wearability: custom-made vs self-adapted mouthguards. J Am Dent Assoc 1994; 125:1112-7.

6 Godwin WC, Craig RG. Stress transmitted through mouth protectors. J Am Dent Assoc 1968;77:1316-20.

7 Hickey JC, Morris AL, Carlson LD, et al. The relation of mouth protectors to cranial pressure and deformation. J Am Dent Assoc 1967;74:735.

8 Patrick DG, van Noort R, Found MS. Evaluation of laminated structures for sports mouthguards. Key Eng Mater 2002;221-222:133-44.

9 Patrick DG, van Noort R, Found MS. The influence of heat treatment on the impact performance of sports mouthguard materials. In: Bandyopadhyay S, ed. Proceedings of ACUN-4 "Composite Systems - Macrocomposites, Microcomposites, Nanocomposites", UNSW, Sydney, Australia, 21-25 July 2002. Sydney, Australia: University of New South Wales, 2002 (ISBN 07334 $18627)$.

10 Hoffmann J, Alfter G, Rudolph NK, et al. Experimental comparative study of various mouthguards. Endod Dent Traumatol 1999;15:157-63.

11 Oikarinen KS, Salonen MAM, Korhonen J. Comparison 238 of the guarding capacities of mouth protectors. Endod Dent Traumatol 1993;9:115-9.

12 Kim HS, Mathieu K. Application of laminates to mouthguards: finite element analysis. J Mater Sci Mater Med 1998;9:457-62.

13 Bulsara YR, Matthew IR. Forces transmitted through a laminated mouthguard material with a Sorbothane insert. Endod Dent Traumatol 1998;14:45-7.

14 Greasley A, Karet B. Towards the development of a standard test procedure for mouthguard assessment. Br J Sports Med 1997;31:31-5.

15 Greasley A, Imlach G, Karet B. Application of a standard test to the in vitro performance of mouthguards. Br J Sports Med 1998;32:17-9.

16 Westerman B, Stringfellow PM, Eccleston JA. Forces transmitted through EVA mouthguard materials of different types and thickness. Aust Dent $J$ 1995;40(6):389-91.

17 Westerman B, Stringfellow PM, Eccleston JA. An improved mouthguard material. Aust Dent J 1997;42(3):189-91.

18 Going RE, Loehman RE, Chan MS. Mouth guard materials: their physical and mechanical properties. J Am Dent Assoc 1974;89:132-8.

19 Scott J, Burke FJT, Watts DC. A review of dental injuries and the use of mouthguards in contact team sports. Br Dent J 1994;176:310-4.

20 Found MS, Howard IC, Paran AP. Interpretation of signals from dropweight impact tests. Composite Structures 1998;42:353-63.

21 Bishop BM, Davies EH, von Fraunhofer JA. Materials for mouth protectors. J Prosthet Dent 1985;53(2):256-61.

\section{bmjupdates+}

bmjupdates+ is a unique and free alerting service, designed to keep you up to date with the medical literature that is truly important to your practice.

bmjupdates+ will alert you to important new research and will provide you with the best new evidence concerning important advances in health care, tailored to your medical interests and time demands.

Where does the information come from?

bmjupdates+ applies an expert critical appraisal filter to over 100 top medical journals A panel of over 2000 physicians find the few 'must read' studies for each area of clinical interest

Sign up to receive your tailored email alerts, searching access and more...

www.bmjupdates.com 